hep-ph/9808419

KIAS-P98018

YUMS 98-016

SNUTP 98-094

Brown-HET-1138

\title{
Neutrino Oscillations and Lepton Flavor Mixing
}

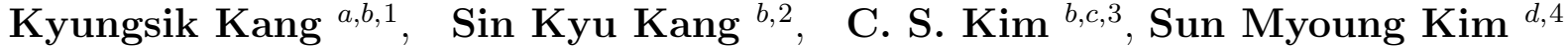 \\ $a$ : Department of Physics, Brown University, Providence, RI 02902, U.S.A. \\ $b$ : School of Physics, Korea Institute for Advanced Study, Seoul 130-012, Korea \\ $c$ : Physics Department, Yonsei University, Seoul 120-749, Korea \\ $d$ : Halla Institute of Technology, Wonju, Korea
}

(July 24, 2021)

\begin{abstract}
In view of the recent announcement on non-zero neutrino mass from Super-Kamiokande experiment, it would be very timely to investigate all the possible scenarios on masses and mixings of light neutrinos. Recently suggested mass matrix texture for the quark CKM mixing, which can be originated from the family permutation symmetry and its suitable breakings, is assumed for the neutrino mass matrix and determined by the four combinations of solar, atmospheric and LSND neutrino data and cosmological hot dark matter bound as input constraints. The charged-lepton mass matrix is assumed to be diagonal so that the neutrino mixing matrix can be identified directly as the lepton flavor mixing matrix and no CP invariance violation originates from the leptonic sector. The results favor hierarchical patterns for the neutrino masses, which follow from the case when either solar-atmospheric data or solar-HDM constraints are used.
\end{abstract}

PACS number(s): 14.60Pq, 12.15Fk

\footnotetext{
${ }^{1}$ kang@het.brown.edu, kskang@kiasph.kaist.ac.kr

${ }^{2}$ skkang@kiasph.kaist.ac.kr, skkang@supy.kaist.ac.kr

${ }^{3}$ kim@cskim.yonsei.ac.kr, http://phya.yonsei.ac.kr/ cskim/

${ }^{4}$ skim@hit.halla.ac.kr
} 
Although neutrino mass is predicted to be zero within the minimal Standard Model, the solar [1, 2] and atmospheric neutrino [2, 3] observations and the Liquid Scintillator Neutrino Detector (LSND) experiment [4] provide likely evidence that neutrinos may have nonzero masses and oscillate. A variety of massive neutrino scenarios [5, 6, 7, 8, 9] have been proposed so as to accommodate the above experimental observations. Among them, the works [6, 7] that use the mass matrix ansatz [10] based on symmetry principle have attracted much attention. Mass matrix ansatz, in fact, has been assumed to predict the entire CabibboKobayashi-Maskawa (CKM) matrix in the quark sector, but most of the earlier works are ruled out [1] because they used the simplest texture for the quark mass matrix and could not predict a heavy top quark mass as measured at Tevatron [12].

Recently, a new class of quark mass matrices [13, 14] has been suggested so that it can be compatible with the measured top quark mass and the measured values of the CKM matrix elements. Its specific form is given by

$$
M_{H}=\left(\begin{array}{ccc}
0 & A & 0 \\
A & D & B \\
0 & B & C
\end{array}\right)
$$

In this paper, we would like to examine if such a type of mass matrix can be suitable for the neutrino sector and accommodate the experimental and/or cosmological observations of neutrino. The form of neutrino mass matrix, in general, needs not be the same as that of charged lepton mass matrix.

We propose that charged lepton mass matrix is taken to be diagonal, whereas neutrino mass matrix is given by the form Eq.(1). Then, the flavor-mixing CKM matrix in the leptonic sector becomes coincident with the neutrino mixing matrix, and CP violation phase can be rotated away in the Yukawa couplings. Very recently, the authors of Ref. 15 considered similar ansatz in view of convenience of diagonalizing mass matrices. However, we would like to stress that this is another new ansatz in which the CP invariance can naturally be imposed to the leptonic sector.

Following the procedure given in Ref. 13], the neutrino mass matrix, which respects in general calculability of the flavor mixing matrix, can be written in terms of neutrino mass eigenvalues $m_{i}$ :

$$
M_{r}^{\nu}=\left(\begin{array}{ccc}
0 & \sqrt{\frac{m_{1} m_{2} m_{3}}{m_{3}-\epsilon}} & 0 \\
\sqrt{\frac{m_{1} m_{2} m_{3}}{m_{3}-\epsilon}} & m_{2}-m_{1}+\epsilon & w\left(m_{2}-m_{1}+\epsilon\right) \\
0 & w\left(m_{2}-m_{1}+\epsilon\right) & m_{3}-\epsilon
\end{array}\right),
$$

where the parameters $\epsilon$ and $w$ are related to each other, and the definition of parameter $w$ is given in Ref. [13]. Confronting the quark mass matrix ansatz as given by the form (2) 
with the measured values of CKM matrix elements, it turned out that the experimentally allowed range of $w$ is $0.97 \leq|w| \leq 1.87$ in the leading approximation. In our analysis, we assume the same range of $w$ for the neutrinos.

The real matrix $M_{r}^{\nu}$ given by Eq. (1) can be diagonalized by a real orthogonal matrix $R^{\nu}$ so that

$$
R^{\nu} M_{r}^{\nu} \tilde{R}^{\nu}=\operatorname{diag}\left(m_{1},-m_{2}, m_{3}\right)
$$

Then, the explicit form of the lepton flavor mixing matrix, i.e. CKM matrix for leptonic sector $V_{l e p}=R^{\nu}$, is given by

$$
V_{l e p}=R^{\nu}=\left(\begin{array}{ccc}
f_{1} & f_{2} & f_{3} \\
\frac{f_{1} m_{1}}{A} & \frac{f_{2}\left(-m_{2}\right)}{A} & \frac{f_{3} m_{3}}{A} \\
\frac{f_{1} B m_{1}}{\left(A *\left(m_{1}-C\right)\right)} & \frac{f_{2} B m_{2}}{\left(A *\left(m_{2}+C\right)\right)} & \frac{f_{3} B m_{3}}{\left(A *\left(m_{3}-C\right)\right)}
\end{array}\right)
$$

where

$$
\begin{aligned}
f_{1} & =\left[1+\left(m_{1} / A\right)^{2}+\left(B m_{1} /\left(A\left(m_{1}-C\right)\right)\right)^{2}\right]^{-1 / 2}, \\
f_{2} & =\left[1+\left(m_{2} / A\right)^{2}+\left(B m_{2} /\left(A\left(m_{2}+C\right)\right)\right)^{2}\right]^{-1 / 2}, \\
\text { and } \quad f_{3} & =\left[1+\left(m_{3} / A\right)^{2}+\left(B m_{3} /\left(A\left(m_{3}-C\right)\right)\right)^{2}\right]^{-1 / 2} .
\end{aligned}
$$

Here, the parameters $A, B, C$ and $D$ are related to the mass eigenvalues by

$$
\begin{aligned}
C+D & =m_{1}-m_{2}+m_{3}, \\
A^{2}+B^{2}-C D & =m_{1} m_{2}+m_{2} m_{3}-m_{3} m_{1} \\
\text { and } A^{2} C & =m_{1} m_{2} m_{3} .
\end{aligned}
$$

Now, we are ready to determine the elements of the lepton mixing (CKM) matrix $V_{l e p}$ from the experimental constraints of solar, atmospheric and LSND neutrino observations as well as cosmological bound. Followings are the experimental constraints on neutrino masses and mixings:

- (A) The solar neutrino deficitf can be explained through the MSW mechanism [16] if $4 \times 10^{-6} \leq \Delta m_{\text {solar }}^{2} \leq 1.2 \times 10^{-5} \mathrm{eV}^{2}$ and $3 \times 10^{-3} \leq \sin ^{2} 2 \theta_{\text {solar }} \leq 1.1 \times 10^{-2}$ (small angle case), or $8 \times 10^{-6} \leq \Delta m_{\text {solar }}^{2} \leq 3 \times 10^{-5} \mathrm{eV}^{2}$ and $0.42 \leq \sin ^{2} 2 \theta_{\text {solar }} \leq 0.74$ (large angle case), and through the just-so vacuum oscillations if $\Delta m_{\text {solar }}^{2} \simeq 10^{-10} \mathrm{eV}^{2}$ and $\sin ^{2} 2 \theta_{\text {solar }} \geq 0.7$ [17].

\footnotetext{
${ }^{5}$ Through out this paper, we will take the small mixing MSW solution for extracting the neutrino mass eigenvalues and lepton flavor mixing parameters.
} 
- (B) The atmospheric neutrino deficit can be accommodated if $0.0005 \leq \Delta m_{\text {atm }}^{2} \leq$ $0.006 \mathrm{eV}^{2}$ and $0.82 \leq \sin ^{2} 2 \theta_{\text {atm }} \leq 1.0$ [18].

- (C) The LSND data indicates $0.27 \leq \Delta m_{L S N D}^{2} \leq 10 \mathrm{eV}^{2}$ and $0.05 \leq \sin ^{2} 2 \theta_{L S N D} \leq 1.0$ 田.

- (D) On the other hand, if light massive neutrinos provide the hot dark matter (HDM) of the Universe, one has to impose $\sum\left|m_{\nu_{i}}\right| \sim 6 \mathrm{eV}$.

- We note that if we consider three generations of neutrino, those results (A-C) can be interpreted by $\nu_{e} \rightarrow \nu_{\mu}, \nu_{\mu} \rightarrow \nu_{\tau}$ and $\bar{\nu}_{\mu} \rightarrow \bar{\nu}_{e}$ oscillations for solar, atmospheric neutrinos and the LSND experiment, respectively . As is well known, it is impossible to construct the lepton flavor mixing matrix in the three-generation neutrino scenario that accommodates all (A-D) of the above three neutrino anomalies and cosmological constraint simultaneously. Thus, one has to sacrifice one or two conditions among the above four results to make the best possible combinations to be considered.

Although there are many possible combinations in three generation light neutrino scenario, in this paper based on the observed fermion mass hierarchy and the possible fermion mass degeneracy, we take only four combinations of neutrino data that would be the most interesting and distinctive:

- (I) solar-atmospheric-HDM neutrino,

- (II) solar-atmospheric neutrino,

- (III) solar-HDM neutrino,

- (IV) atmospheric-LSND neutrino.

We note that those four cases fall into two categories. One is almost degenerate threeneutrino scenario and the other is hierarchical neutrino scenario. Almost degenerate threeneutrino scenario can be achieved by choosing the neutrino data set (I), while the hierarchical neutrino structures are led to by the sets (II), (III) and (IV). Notice that the sets (II) and (III) have hierarchical structure $m_{1}<<m_{2}<<m_{3}$, while the set (IV) has $m_{1}<<m_{2} \sim m_{3}$. We also note that the other possible combinations give not much different physics results from the above four cases (I-IV).

\footnotetext{
${ }^{6}$ The recent experiment by CHOOZ [19] disfavors $\nu_{\mu} \rightarrow \nu_{e}$ oscillation for atmospheric neutrinos. In addition, more recent result from KARMEN2 seems to have excluded most of the region suggested by the LSND experiment [20].
} 
From the constraints of neutrino data sets (I) - (IV), one can easily extract the appropriate eigenvalues of neutrino masses $m_{\nu_{i}}$ :

(I) solar-atmospheric-HDM

$$
m_{\nu_{1}}=1.9979-1.9996, \quad m_{\nu_{2}}=1.9979-1.99996, \quad m_{3}=2.00084-2.0042 \quad(\mathrm{eV})
$$

(II) solar-atmospheric

$$
m_{\nu_{1}} \sim 0, \quad m_{\nu_{2}} \sim 10^{-2}-10^{-3}, \quad m_{\nu_{3}} \sim 0.0717 \quad(\mathrm{eV})
$$

(III) solar-HDM

$$
m_{\nu_{1}} \sim 10^{-5}, \quad m_{\nu_{2}} \sim 10^{-2}, \quad m_{\nu_{3}} \sim 3 \quad(\mathrm{eV})
$$

(IV) atmospheric-LSND

$$
m_{\nu_{1}} \sim 0.5 \times 10^{-5}, \quad m_{\nu_{2}} \sim 0.52, \quad m_{\nu_{3}} \sim 0.524 \quad(\mathrm{eV})
$$

Note that we have not introduced any specific model in Eqs. (4-7), which are most reasonable and distinctive based on items (A-D), as explained before.

Following the numerical analysis of Eqs. (2-3) and using the above neutrino mass eigenvalues (4-7), we can obtain the following lepton flavor mixing (CKM) matrices respectively:

$$
\begin{array}{rlrl}
\text { (I) } & V_{\text {lep }}= & =\left(\begin{array}{ccc}
0.6925 & 0.7071 & 0.1433 \\
0.6924 & -0.7071 & 0.1434 \\
0.2027 & -0.00006 & -0.9792
\end{array}\right) \\
\text { (II) } & V_{\text {lep }}= & \left(\begin{array}{ccc}
0.9950 & 0.0995 & 0.0000 \\
0.0729 & -0.7293 & 0.6803 \\
-0.0677 & 0.6769 & 0.7330
\end{array}\right) \\
\text { (III) } & V_{\text {lep }}=\left(\begin{array}{ccc}
0.9950 & 0.0995 & 0.0000 \\
0.0729 & -0.7292 & 0.6804 \\
-0.0677 & 0.6770 & 0.7328
\end{array}\right) \\
\text { (IV) } & V_{\text {lep }}= & \left(\begin{array}{ccc}
0.9998 & 0.0198 & 0.0086 \\
0.0147 & -0.9164 & 0.3999 \\
0.0158 & -0.3997 & -0.9165
\end{array}\right)
\end{array}
$$

Now, let us discuss if those obtained lepton flavor mixing (CKM) matrices, Eqs. (8-11), can be compatible with the constraints of neutrino experiments (items A-D). The mixing parameters $\sin ^{2} 2 \theta$ for the solar, atmospheric and LSND neutrino oscillations can be related to 24

$$
\begin{aligned}
\sin ^{2} 2 \theta_{\text {solar }} & \approx 4\left|V_{e 1}\right|^{2}\left|V_{e 2}\right|^{2}, \\
\sin ^{2} 2 \theta_{\text {atm }} & \approx 4\left|V_{\mu 3}\right|^{2}\left|V_{\tau 3}\right|^{2}, \\
\text { and } \sin ^{2} 2 \theta_{L S N D} & \approx 4\left|V_{e 3}\right|^{2}\left|V_{\mu 3}\right|^{2} .
\end{aligned}
$$


- This case $(\mathrm{I})$ predicts $\sin ^{2} 2 \theta_{\text {solar }} \approx 0.969$ which implies maximal mixing between $\nu_{e}$ and $\nu_{\mu}$ and small mixing between $\nu_{\mu}$ and $\nu_{\tau}$. This mixing pattern is ruled out because the recent CHOOZ and Super-Kamiokande experiments seem to disfavor $\nu_{\mu} \rightarrow \nu_{e}$ oscillation for atmospheric neutrino with a large mixing. Thus, almost degenerate three-neutrino scenario can not be achieved in our scheme.

- The flavor mixing matrix (II) leads to $\sin ^{2} 2 \theta_{\text {solar }} \approx 0.039$ and $\sin ^{2} 2 \theta_{\text {atm }} \approx 0.995$ which are consistent with the recent experimental results for solar and atmospheric neutrino anomalies, which can be interpreted as neutrino oscillations.

- The case (III) shows similar pattern of mixing matrices as in (II), but predicts somewhat different mixing angle between $\nu_{\mu}$ and $\nu_{\tau}$ from the case (IV).

- This mixing matrix (IV) leads to $\sin ^{2} 2 \theta_{\text {atm }} \approx 0.537$ and $\sin ^{2} 2 \theta_{L S N D} \approx 0.0005$ which can not be compatible with the results for neutrino oscillations from atmospheric neutrino and LSND experiment.

Let us check if our neutrino mass matrix ansatz can be free from the constraints on neutrinoless double beta decay. As is well known, nonobservation of the neutrinoless double beta decay provides neutrino mass bound, $<m_{\nu_{e}}>=\left|\sum_{i=1}^{3} \eta_{i} V_{\nu_{e}}^{2} m_{i}\right| \leq 0.45 \mathrm{eV}^{2}$ [21]. If we impose the CHOOZ and Bugey results [22], $<m_{\nu_{e}}>\leq 3 \times 10^{-2} \mathrm{eV}$ [23]. As can be easily expected from our mixing matrices (II)-(III), our numerical results lie below these bounds.

In view of recent announcement non-zero neutrino masses from Super-Kamiokande experiment, it would be very timely to investigate all the possible scenarios on masses and mixings of three light neutrinos. Starting from phenomenological four texture zeros lepton mass matrices, which can be originated from the family permutation symmetry and its suitable breakings, we investigated the physical consequences by comparing our theoretical predictions with the solar and atmospheric neutrino observations, the LSND experiment as well as the hot dark matter bound. We found that our scheme favors hierarchical patterns for the neutrino masses, which follow from the case when either solar-atmospheric data or solar-HDM constraints are used. 


\section{Acknowledgments}

Two of us (KK and CSK) wish to thank the Korea Institute for Advanced Study for warm hospitality. The work of KK is supported in part by the US DOE contract DE-FG02-91ER40688-Task A. The work of CSK was supported in part by Non-Directed-Research-Fund made in the program year of 1997, in part by the CTP, Seoul National University, in part by the BSRI Program, Ministry of Education, Project No. BSRI-98-2425, and in part by the KOSEF-DFG large collaboration project, Project No. 96-0702-01-01-2. 


\section{References}

[1] B. T. Cleveland et al., Nucl. Phys. B(Proc. Suppl.) 38, 47 (1995); K. S. Hirata et al., Phys. Rev. 44, 2241 (1991); GALLEX Collab., Phys. Lett. B 388, 384 (1996); J. N. Abdurashitov et al., Phys. Rev. Lett. 77, 4708 (1996).

[2] Y. Suzuki, Invited talk at Erice Neutrino Workshop, September 17-22, 1997.

[3] K. S. Hirata et al., Phys. Lett. B 280, 146 (1992); R. Becker-Szendy et al., Phys Rev. D 46, 3720 (1992); W. W. M. Allison et al., Phys. Lett. B 391, 491 (1997); Y. Fukuda et al., Phys. Lett. B 335, 237 (1994).

[4] C. Athanassopoulos et al., Phys. Rev. Lett. 75, 2650 (1995); nucl-ex/9706006.

[5] D. O. Caldwell and R. N. Mohapatra, Phy. Rev. D 48, 3259 (1993); S. T. Petcov and A. Smirnov, Phys. Lett. B 322, 109 (1994); A. S. Joshipura, Z. fur Phys. C 64, 31 (1994); A. Ionissian and J. W. F. Valle, Phys. Lett. B 332, 93 (1994); P. Bamert and C. P. Burgess, Phys. Lett. B 329, 289 (1994); D. G. Lee and R. N. Mohapatra, Phys. Lett. B 329, 463 (1994); R. N. Mohapatra and S. Nussinov, Phys. Lett. B 346, 75 (1995); P. Harrison, D. Perkins and W. Scott, Phys. Lett. B 349, 137 (1995); A. Acker and S. Pakvasa, Phys. Lett. B 397, 209 (1997); P. Krastev and S. Petcov, Phys. Lett. B 395, 69 (1997); J. Peltoniemi and J. W. F. Valle, Nucl. Phys. B 406; S. M. Bilenky, C. Giunti and W. Grimus, hep-ph/9607372; hep-ph/9711311; C. Cardall and G. Fuller, astro-ph/9606024; C. Cardall, D. Cline and G. Fuller, hep-ph/9706426.

[6] K. Kang, J. E. Kim and P. Ko, Z. Phys. C 72, 671 (1996); K. Kang, S. K. Kang, J. E. Kim and P. Ko, Mod. Phys. Lett. A12, 1175 (1997); K. Kang and S. K. Kang, hep-ph/9802328.

[7] H. Fritzsch and Z. Xing, Phys. Lett. B 372, 265 (1996); hep-ph/9808272.

[8] Z. Xing, hep-ph/9804433; V. Barger, T. J. Weiler and K. Whisnant, hep-ph9807319; H. Georgi and S. L. Glashow, hep-ph/9808293; R. Barbieri, L. J. Hall and A. Strumia, hep-ph/9808333; M. Fukugita, M. Tanimoto and T. Yanagida, Phys. Rev. bf D57, 4429 (1998); M. Tanimoto, hep-ph/9807517 and references therein.

[9] C. S. Kim and G. Cvetic, Talk given at MMQL97, Shizuoka, Japan (hep-ph/9706451); G. Cvetic and C. S. Kim, Phys. Rev. D51, 201 (1996); Nucl. Phys. B407, 290 (1993); Mod. Phys. Lett. A9, 289 (1994); Int. J. Mod. Phys. A9, 1495 (1994). 
[10] Rothman and K. Kang, Phys. Rev. Lett. 43, 1548 (1979); H. Fritzsh, Phys. Lett. 73, 317 (1978); Nucl. Phys. B 155, 189 (1979)

[11] K. Kang and S. Hadjitheodoridis, Phys. Lett. B 193, 504 (1987); H. Harari and Y. Nir, Phys. Lett. B 195, 586 (1987); K. Kang, J. Flanz and E. Paschos, Z. Phys. C55, 75 (1992).

[12] CDF Collab., F. Abe et al., Phys. Rev. Lett. 74, 2676 (1995); D0 Collab., A. Abachi et al., Phys. Rev. Lett. 74, 2636 (1995).

[13] K. Kang and S. K. Kang, Phys. Rev. D 56, 1511 (1997).

[14] See also, P. Kaus and S. Meshkov, Phys. Rev. D 42, 1863 (1990); F. Cuypers and C. S. Kim, Phys. Lett B254, 462 (1991); H. Fritzsch and Z. Xing, Phys. Lett. 353, 114 (1995); ibid, 413, 396 (1997); H. Lehmann, C. Newton and T. T. Wu, Phys. Lett. B 384, 249 (1996); Z. Xing, hep-ph/9609204; P. S. Gill and M. Gupta, J. Phys. G 23, 335 (1997); Phys. Rev. D 56, 3143 (1997); S. L. Adler, hep-ph/9711393; hep-ph/9806518;

A. Mondragon, E. Rodriguez-Jauregui, hep-ph/9804267; hep-ph/9807214.

[15] P. S. Gill and M. Gupta, Phys. Rev. D 57, 3971 (1998).

[16] L. Wolfenstein, Phys. Rev. D 17, 2369 (1978); S. P. Mikheyev and A. Smirnov, Yad. Fiz. 42, 1441 (1985); Nuovo Cimento 9 C, 17 (1986).

[17] G. L. Fogli, E. Lisi and D. Montanino, Astropart. Phys. 9, 119 (1998).

[18] Y. Fukuda et al., Super-Kamiokande Collaboration, "Evidence for oscillation of atmospheric neutrinos", to appear in Phys. Rev. Lett., hep-ex/9807003.

[19] M. Apollonio et al. hep-ex/9711002.

[20] B. Zeitnitz, talk given at the Neutrino98, Takayama, Japan, June 1998.

[21] Heidelberg-Moscow Collab., Phys. Lett. B 407, 219 (1997) ; H.V. KlapdorKleingrothaus et al., hep-ph/9712381.

[22] B. Achkar et al., Nucl. hys. B 434, 503 (1995)

[23] S. M. Bilenky, C. Giunti, C. W. Kim and M. Monteno, hep-ph/9711400.

[24] S. M. Bilenki, A. Bottino, C. Giunti and C. W. Kim, hep-ph/9512353; S. M. Bilenki and C. Giunti, hep-ph/9802201. 